\title{
Drag force on heavy diquarks and gauge/string duality
}

\author{
Oleg Andreev \\ L. D. Landau Institute for Theoretical Physics, Kosygina 2, 119334 Moscow, Russia \\ and Arnold Sommerfeld Center for Theoretical Physics, \\ LMU-München, Theresienstrasse 37, 80333 München, Germany
}

(Received 3 May 2018; published 14 September 2018)

\begin{abstract}
We use gauge/string duality to model a doubly heavy diquark in a color antitriplet moving in a thermal plasma at temperatures near the critical. With the assumption that there is no relative motion between the constituents, we calculate the drag force on the diquark. At high enough speed we find that diquark string configurations develop a cusp. In addition, we estimate the spatial string tension at nonzero baryon chemical potential, and also briefly discuss an extension to a triply heavy triquark in a color triplet.
\end{abstract}

DOI: $10.1103 /$ PhysRevD.98.066007

\section{INTRODUCTION}

The concept of a diquark emerged quite naturally and inescapably as an organizing principle for hadron spectroscopy [1]. ${ }^{1}$ Actually, it originates from the fact that in QCD, a strong force between quarks in a color antitriplet channel $\overline{\mathbf{3}}$ is attractive. Since diquarks may be very useful degrees of freedom to focus on in QCD, it is of great importance to study them in a pure and isolated form and determine their parameters. The latter is not straightforward because of confinement, since diquarks are colored. Of course, the same problem arises for quarks. A possible way out is to do so in the deconfined phase that allows one to study them in isolation, but for temperatures above the critical (pseudocritical) temperature $T_{c}$. This works for quarks regardless of how high temperature is, but it becomes more tricky for diquarks. The situation here is quite similar to that of $J / \Psi$ mesons. The point is that diquarks as bound states dissolve at temperatures above the dissociation temperature $T_{\text {diss }}$. The numerical analysis shows that for bottomonium states $T_{\text {diss }}$ is compatible with $2 T_{c}$ [3]. This opens a narrow window of opportunity for studying heavy diquarks in isolation.

One of the profound implications of the AdS/CFT correspondence for QCD is that it rekindled interest in finding its string description (string dual). This time the main efforts have been focused on a five (ten)-dimensional string theory in a curved space rather than on an old-fashioned

\footnotetext{
${ }^{1}$ For recent developments, see Ref. [2] and numerous references therein.

Published by the American Physical Society under the terms of the Creative Commons Attribution 4.0 International license. Further distribution of this work must maintain attribution to the author(s) and the published article's title, journal citation, and DOI. Funded by SCOAP ${ }^{3}$.
}

four-dimensional theory in Minkowski space. In this paper we continue these efforts. Our goal here is quite specific: to see what insight can be gained into doubly heavy diquarks moving in a hot medium by using five-dimensional effective string models. The main reasons for doing this are twofold: (1) Because a string dual to QCD is not known at the present time. So, it is worth gaining some experience by solving any problems that can be solved within the effective string models already at our disposal. (2) Because, to our knowledge, this issue has never been discussed in the existing literature, even that related to hadron phenomenology and lattice QCD.

To contrast, what has been widely discussed is the motion of a heavy quark in a hot medium (thermal plasma) [4]. In the Langevin formalism, the motion is described by a stochastic differential equation

$$
\frac{d \mathbf{p}}{d t}=-\eta_{\mathrm{D}} \mathbf{p}+\boldsymbol{\xi}(t)
$$

such that the right-hand side is written as the sum of a drag force, with $\eta_{\mathrm{D}}$ being a drag coefficient, and a random force. This also attracted much attention and became a hot topic in the context of AdS/CFT [5]. In particular, in Ref. [6] it was proposed how to calculate the drag coefficient by just considering a trailing string, as sketched in Fig. 1. Importantly enough, for more realistic string models, such a proposal gives reasonable estimates for the heavy quark diffusion coefficients which are compatible with those of lattice QCD [7].

The paper is organized as follows: In Sec. II, we begin by setting the framework and recalling some preliminary results on string configurations for diquarks. Then, we show how to calculate the drag force on a doubly heavy diquark moving in a hot medium. In Sec. III, we consider a concrete example to demonstrate how the proposal might work in practice. Some of the more technical details are 

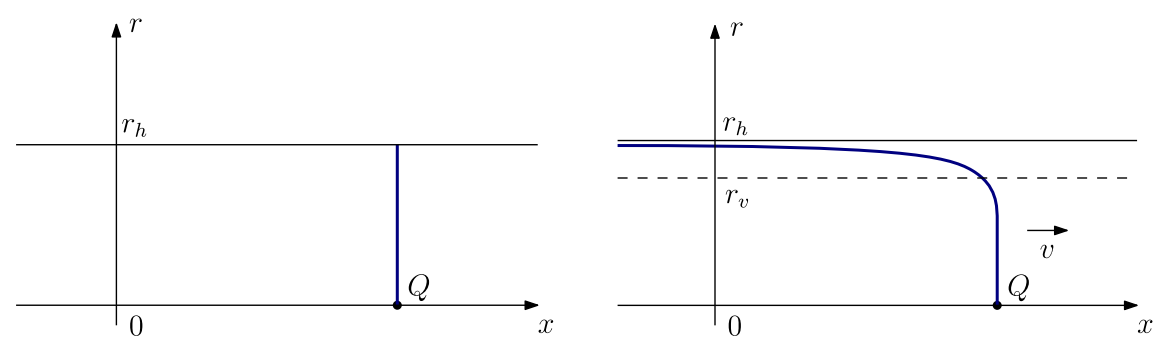

FIG. 1. (Left) A string attached to a static quark $Q$ at a point on the boundary of AdS space and terminated at the black hole horizon, located at $r=r_{h}$ in the interior. (Right) A string trailing out from a quark moving with speed $v$. The dashed line indicates the induced horizon related to an induced metric on the string world sheet, as explained in Appendix A.

given in the appendixes. We conclude in Sec. IV with a discussion of several open problems that we hope will stimulate further research.

\section{DRAG FORCE ON DOUBLY HEAVY DIQUARKS AS SEEN BY STRING THEORY}

\section{A. Preliminaries}

For orientation, we begin by setting the framework and recalling several preliminary results. We will follow the strategy of calculating the drag force by means of a fivedimensional effective string theory that is nowadays quite common for modeling QCD at finite temperature. A key point is that the background geometry is that of a black hole. The latter implies that a dual gauge theory is in the deconfined phase.

The strings in question are Nambu-Goto strings governed by the action $S=-\frac{1}{2 \pi \alpha^{\prime}} \int d \tau d \sigma \sqrt{-\gamma}$ and living in a five-dimensional curved space with a metric of form

$$
d s^{2}=w(r) R^{2}\left(-f(r) d t^{2}+d x_{i}^{2}+f^{-1}(r) d r^{2}\right),
$$

where $x_{i}=(x, y, z)$. The blackening factor $f$ is monotonically decreasing from unity to zero on the interval $\left[0, r_{h}\right]$. One can think of this spacetime as a deformation of the Schwarzschild black hole in $\mathrm{AdS}_{5}$ space such that the boundary is at $r=0$ and the horizon at $r=r_{h}$. A dimensionful deformation parameter is needed to depart from conformality of $\mathrm{AdS}_{5}$ for the purpose of mimicking infrared properties of QCD. One of the requirements is that the metric approach that of the Schwarzschild black hole with $f=1-\left(\frac{r}{r_{h}}\right)^{4}$ as $r$ approaches the boundary. As in Ref. [7], we assume that this model provides a reasonable approximation to the behavior of QCD in the deconfined phase near the critical (pseudocritical) temperature. Importantly, the temperature of a dual gauge theory is given by the Hawking temperature of the black hole, $T=\frac{1}{4 \pi}\left|\frac{d f}{d r}\right|_{r=r_{h}}$. The thermal medium is assumed to be isotropic, and thus the metric is chosen to be invariant under spatial rotations.

In contrast to the single-quark case, this is not the whole story. The reason is that the stringy construction of diquark states in color antitriplets demands a new object [8]. It is a

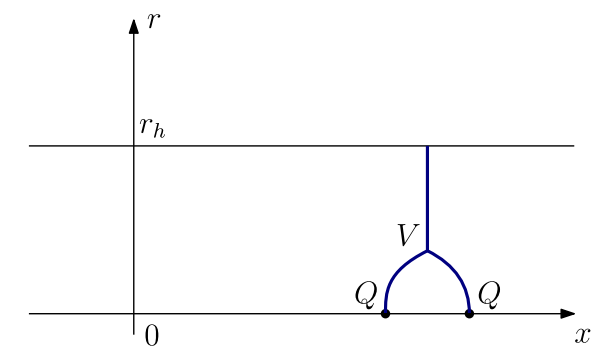

FIG. 2. A static diquark configuration in the color $\overline{\mathbf{3}}$ channel. The heavy quarks $Q$ are placed on the boundary of space. The two strings attached to the quarks and one attached to the horizon join at the baryon vertex $V$ in the interior.

baryon vertex (string junction), at which three strings meet together. In five dimensions, the resulting construction is sketched in Fig. 2. Noteworthily, it leads to a result for the diquark free energy which is consistent with lattice simulations [8].

From a string theory point of view, such a vertex is a five-brane wrapped on a five-dimensional internal manifold in ten-dimensional space [9]. In the above construction we assume that quarks are placed at the same point in the internal space, and therefore, the detailed structure of this space is not important for us, except for a possible warp factor depending on the radial coordinate $r$. Note that the vertex can be regarded as pointlike in five-dimensional space.

Taking the leading term in the $\alpha^{\prime}$ expansion of the worldvolume action for the brane and choosing static gauge results in an effective action for the baryon vertex

$$
S_{\text {vert }}=-m \int d t \sqrt{f} \mathcal{V}(r)
$$

Here $m$ is a parameter which takes account of a volume of the internal space together with possible $\alpha^{\prime}$ corrections in the brane action. ${ }^{2}$ The form of $\mathcal{V}$ is determined by the warp

\footnotetext{
${ }^{2}$ On the way to the string dual to quantum chromodynamics (QCD), $\mathrm{AdS}_{5} \times \mathbf{S}^{5}$ is supposed to be replaced by its deformation, such that $\mathbf{S}^{5} \rightarrow \mathbf{X}$, with $\mathbf{X}$ being a new compact space.
} 


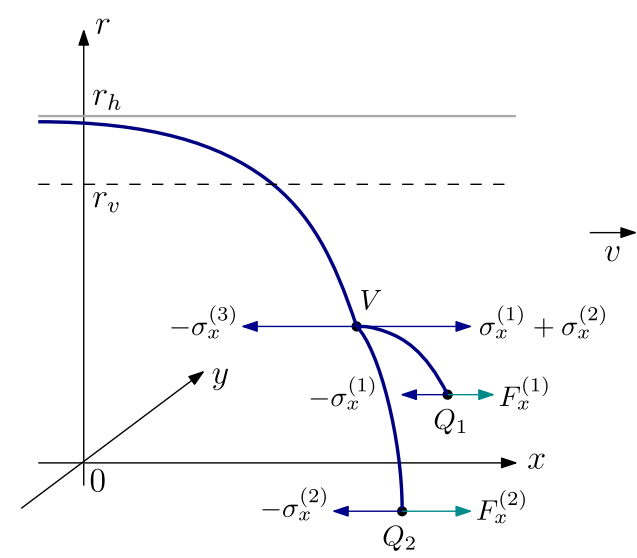

FIG. 3. A diquark configuration moving with speed $v$ in the $x$ direction. The heavy quarks are set on the boundary. $V$ is a baryon vertex located at $r=r_{0}$ in the interior. It is connected to the quarks by the two strings. The remaining third string is trailing out from the vertex. External forces $F_{x}^{(i)}$ exerted on the quarks are needed to balance internal string tensions $\sigma_{i}$.

factor of the metric. It is also worth bearing in mind that $S_{\text {vert }}$ is a function of the radial coordinate $r$.

\section{B. Calculating the drag force}

We now want, as in Fig. 1 for a single quark, to consider a diquark configuration moving with speed $v$. In doing so, we can without loss of generality place heavy quark sources at arbitrary points on the $x-y$ plane, at $r=0$, and consider their uniform motion with speed $v$ in the $x$ direction, as sketched in Fig. 3. We will work in the lab frame related to the thermal medium of a dual gauge theory and assume that there is no relative motion between the quarks.

Let us begin by explaining, in a qualitative way, what one might expect from such a configuration. The argument goes as follows: Since the motion is uniform, nothing happens with the shape of the two strings connected to the quarks. In fact, what the external forces do is that they pull the remaining (trailing) string in the $x$ direction, and this costs energy. Then, the same energy-based argument that one uses in the single-quark case shows that in both cases the drag forces are equal in magnitude. This argument is robust to the variations of string shapes (for the strings terminated on the quarks) and of the form of $\mathcal{V}$.

It is natural to think of the above argument as a starting point for understanding the diquark case. To understand that better, let us translate it into mathematical language. First, for each string we take the static gauge ${ }^{3}$

$$
t(\tau)=\tau, \quad r(\sigma)=a \sigma+b,
$$

where $(\tau, \sigma)$ are world-sheet coordinates. Combining these with the boundary conditions

\footnotetext{
${ }^{3}$ If the context is clear, we sometimes omit the index labeling the strings.
}

$r_{1}(0)=r_{2}(0)=0, \quad r_{1}(1)=r_{2}(1)=r_{3}(0)=r_{0}$,

$r_{3}(1)=r_{h}$,

we get $a_{1}=a_{2}=b_{3}=r_{0}, a_{3}=r_{h}-r_{0}$, and $b_{1}=b_{2}=0$. Then, for each string we choose the ansatz

$$
x(r)=v t+\xi(r), \quad y(r)=\eta(r),
$$

which is a slightly extended version of that originally proposed in Ref. [6].

In the presence of external forces exerted on the quarks, the total action $\mathcal{S}$ is the sum of three Nambu-Goto actions, an effective action of the baryon vertex, and boundary terms arising from the coupling with external fields:

$$
\mathcal{S}=\sum_{i=1}^{3} S_{i}+S_{\mathrm{vert}}+S_{\mathrm{b}}
$$

Taking the metric (2.1) and using the ansatz (2.5), we find that each Nambu-Goto action takes the form

$$
S=-\mathfrak{g} \int d t \int d r w \sqrt{1-\frac{v^{2}}{f}+f\left(\partial_{r} \xi\right)^{2}+\left(f-v^{2}\right)\left(\partial_{r} \eta\right)^{2}},
$$

with $\mathfrak{g}=\frac{R^{2}}{2 \pi \alpha^{\prime}}$. Similarly, for the baryon vertex, taking a fivebrane effective action along with the ansatz $x=v t$, we find that the action (2.2) now becomes

$$
S_{\text {vert }}=-m \int d t \sqrt{f-v^{2}} \mathcal{V}\left(r_{0}\right)
$$

Actually, what we have written above, though incomplete, is enough for our purposes, because we do not need the explicit form of $S_{b}$. We just need the fact that the variation of $\mathcal{S}$ is given by ${ }^{4}$

$$
\begin{aligned}
\delta \mathcal{S}= & -\sum_{i=1}^{3} \int d t \int d r\left(\partial_{r} \sigma_{x}^{(i)} \delta \xi_{i}+\partial_{r} \sigma_{y}^{(i)} \delta \eta_{i}\right) \\
& +\int d t\left(F_{x}^{(1)}-\sigma_{x}^{(1)}\right) \delta x_{1}+\left(F_{x}^{(2)}-\sigma_{x}^{(2)}\right) \delta x_{2} \\
& +\left(\sigma_{x}^{(1)}+\sigma_{x}^{(2)}-\sigma_{x}^{(3)}\right) \delta x_{0}+\cdots,
\end{aligned}
$$

where the dots stand for other boundary contributions. This formula is derived at time $t=0$, when the $x$ coordinates of the quarks and vertex are $x_{i}$ and $x_{0}$, respectively. Since the motion is uniform, all the equations obtained via the variational principle are time independent. Looking at the right-hand side of Eq. (2.9), we can immediately obtain

\footnotetext{
${ }^{4}$ The sign conventions for the $\sigma$ 's and $F$ 's are those of Fig. 3.
} 
the first integrals of the equations of motion: $\sigma_{x}=c$ and $\sigma_{y}=c^{\prime}$, where $c$ and $c^{\prime}$ are constants and

$$
\begin{gathered}
\sigma_{x}=-\frac{\mathfrak{g} w f \partial_{r} \xi}{\sqrt{1-\frac{v^{2}}{f}+f\left(\partial_{r} \xi\right)^{2}+\left(f-v^{2}\right)\left(\partial_{r} \eta\right)^{2}}}, \\
\sigma_{y}=-\frac{\mathfrak{g} w\left(f-v^{2}\right) \partial_{r} \eta}{\sqrt{1-\frac{v^{2}}{f}+f\left(\partial_{r} \xi\right)^{2}+\left(f-v^{2}\right)\left(\partial_{r} \eta\right)^{2}}} .
\end{gathered}
$$

It is easy to write these formulas in another form more convenient for our analysis:

$$
\left(\partial_{r} \xi\right)^{2}=\frac{1}{f} \frac{1-\frac{v^{2}}{f}}{\frac{\bar{\sigma}^{2}}{\sigma_{x}^{2}}-1-\left(1-\frac{v^{2}}{f}\right)^{-1} \frac{\sigma_{y}^{2}}{\sigma_{x}^{2}}}, \quad\left(\partial_{r} \eta\right)^{2}=\frac{\sigma_{y}^{2}}{\sigma_{x}^{2}} \frac{\left(\partial_{r} \xi\right)^{2}}{\left(1-\frac{v^{2}}{f}\right)^{2}},
$$

where $\bar{\sigma}=\mathfrak{g} w \sqrt{f}$ is an effective string tension [10].

Now, let us consider the trailing string. It is helpful to first analyze the case $c^{\prime}=0$. From Eq. (2.10), it follows that $\eta$ is constant. This means that the string lies on the $x-r$ plane. If so, then we can proceed along the lines of Ref. [6], briefly reviewed in Appendix A. The key point is that the only way to have $\left(\partial_{r} \xi\right)^{2}>0$ is to set

$$
\sigma_{x}^{(3)}=\bar{\sigma}_{v},
$$

where $\bar{\sigma}_{v}=\bar{\sigma}\left(r_{v}\right)$, and $r_{v}$ is a solution of the equation $f=v^{2}$. Such a solution always exists, because $f$ monotonically decreases from 1 to 0 on the interval $\left[0, r_{h}\right]$.

Now, taking $c^{\prime} \neq 0$, the function $1-\frac{v^{2}}{f}$ is positive on the interval $\left[0, r_{v}\right]$. The function $\frac{\bar{\sigma}^{2}}{\sigma_{x}^{2}}-1-\left(1-\frac{v^{2}}{f}\right)^{-1} \frac{\sigma_{y}^{2}}{\sigma_{x}^{2}}$ goes to positive infinity as $r$ goes to zero and to negative infinity as $r$ goes to $r_{v}{ }^{5}$ From this, it follows that it has one or more zeros on $\left[0, r_{v}\right]$. If so, then it is negative on the interval between the largest zero and end point $r=r_{v}$. As a result, $\left(\partial_{r} \xi\right)^{2}<0$ on such an interval, and therefore, there is no trailing solution.

The conditions for mechanical equilibrium can be deduced from the vanishing of the boundary terms in the variation of the total action. In particular, Eq. (2.9) implies that

$$
F_{x}^{(1)}-\sigma_{x}^{(1)}=0, \quad F_{x}^{(2)}-\sigma_{x}^{(2)}=0
$$

for the quarks, and

$$
\sigma_{x}^{(1)}+\sigma_{x}^{(2)}-\sigma_{x}^{(3)}=0
$$

for the vertex. From these formulas, it follows that the total force exerted on the diquark is $F_{\text {tot }}=F_{x}^{(1)}+F_{x}^{(2)}=\sigma_{x}^{(3)}$,

\footnotetext{
${ }^{5}$ Note that the effective string tension $\bar{\sigma}$ for $r \rightarrow 0$ behaves as $\bar{\sigma} \sim 1 / r^{2}$.
}

with $\sigma_{x}^{(3)}$ given by Eq. (2.12). Using the fact that $F_{\text {drag }}=-F_{\text {tot }}$, we get

$$
F_{\text {drag }}=-\bar{\sigma}_{v}
$$

We have obtained precisely the result, as anticipated before. It can be written more explicitly as $F_{\text {drag }}=-\mathfrak{g} w\left(r_{v}\right) v$. The same expression was also found in Ref. [11] for a single quark. We have thus shown that if there is no relative motion between the quarks, then the drag force on a diquark is equal to that on a single quark.

\section{A CONCRETE EXAMPLE}

In Sec. II, we obtained the formula for the drag force acting on a doubly heavy diquark without paying much attention to the problem of existence of the corresponding string configuration. Now, we will to some extent settle the problem using a simple example. Once we do this, we find a rather nontrivial structure of the diquark configuration even in that case. In particular, a cusp develops on one of the strings at high enough speed.

\section{A. The model}

For the warp and blackening factors, we set

$$
w(r)=\frac{\mathrm{e}^{\mathfrak{b} r^{2}}}{r^{2}}, \quad f(r)=1-\left(\frac{r}{r_{h}}\right)^{4},
$$

which can be obtained from the Euclidean metric of Ref. [12] by the standard Wick rotation $t \rightarrow i t$. One might think of criticizing such a choice on the grounds of the consistency of the string sigma model at the loop level, but there is not any restriction at the classical level where we are working. The advantages are twofold. First, it allows a great deal of simplification of the resulting equations, which makes it useful for understanding more complicated forms of $w$ and $f$. Second, it provides quite plausible results for $S U(3)$ pure gauge theory, which are in some cases amazingly close to those of lattice gauge theory [13].

Given the explicit form of $f$, it is straightforward to determine the Hawking temperature as a function of $r_{h}$, and also find the position of the induced horizon

$$
T=\frac{1}{\pi r_{h}}, \quad r_{v}=r_{h} \sqrt[4]{1-v^{2}} .
$$

We also take note of the fact that with the above choices for the warp and blackening factors, the critical temperature is given by $T_{c}=\frac{\sqrt{2}}{\pi}$ [12]. From this formula and Eq. (3.2), it is found convenient to introduce a dimensionless parameter $h=\mathfrak{s} r_{h}^{2}=\frac{T_{c}^{2}}{T^{2}}$. So, $h<1$ in the deconfined phase.

We complete the model by specifying the gravitational potential for the vertex. Following Ref. [14], we take it to be of the form 


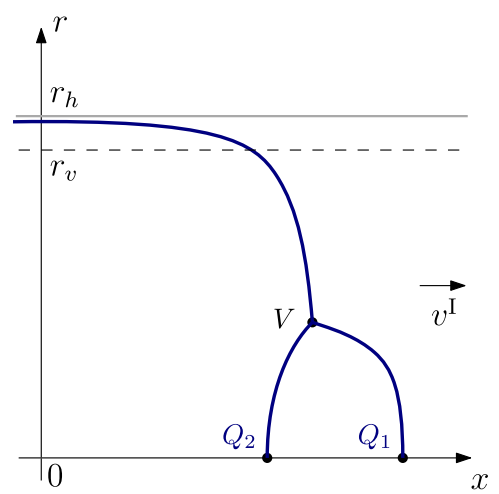

(I)

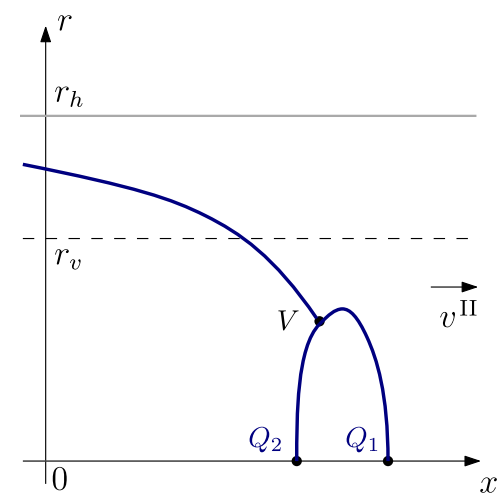

(II)

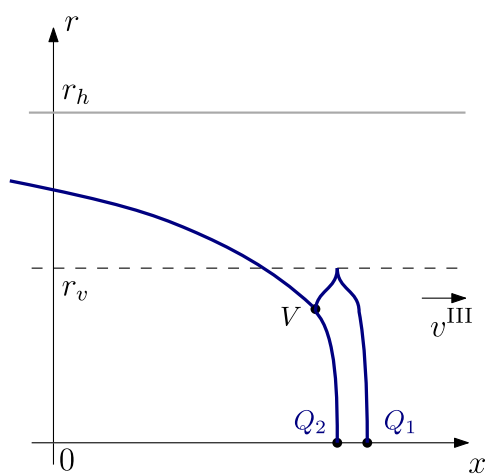

(III)

FIG. 4. A planar diquark configuration moving in the $x$ direction. Here the $r$ coordinate of the vertex is fixed at $r_{0}$, while $v$ takes different values such that $v^{\mathrm{I}}<v^{\mathrm{II}}<v^{\mathrm{III}}$. In the case III, a cusp forms at a point lying on the induced horizon.

$$
\mathcal{V}(r)=\frac{\mathrm{e}^{-2 \mathfrak{s} r^{2}}}{r} .
$$

Apart from the brane construction, another crucial reason for this is the ability of the model to describe the lattice data for the three-quark potentials [14].

\section{B. A diquark configuration moving along the quark-quark axis}

As an example, we consider a diquark moving at a constant speed along its axis. Our aim is twofold: first, to address the problem of existence of the diquark configuration, and second, to see what kind of length contraction one could expect in the model under consideration. Of course, this cannot be the whole story because of the diquark's ability to move in an arbitrary direction with respect to the axis. The latter greatly adds to the complexity of the problem but would not affect our main conclusions, as we expect based on the results for a quark-antiquark pair in which the direction of the hot wind has only a little effect [5].

We take, without loss of generality, the diquark axis to be the $x$ axis. Since the speed of the diquark is directed in the $x$ direction, on symmetry grounds, the corresponding string configuration is planar. It lies on the $x-r$ plane, as sketched in Fig. 4. In this figure, we do not indicate the external forces and internal string tensions explicitly. Like in the previous section, we work in the lab frame and assume that the quark relative speed is zero.

\section{Configuration I}

Consider the static configuration sketched in Fig. 2. To get further, we need to boost it along the $x$ axis with a small speed $v$. In this case, we can get a good idea of what to expect based on the experience gained from the trailing string picture shown in Fig. 1. Thus, it seems reasonable that very little happens with the strings terminated on the quarks, while the string previously terminated on the horizon drastically changes its shape and becomes a trailing string. At late times the resulting configuration will be like that shown in Fig. 4 on the left.

Since the configuration moves uniformly, we can invoke the formula (A11) to write the distance between the quarks as

$$
\ell(\nu ; v, h)=\sqrt{\frac{h}{\mathfrak{G}}} \int_{0}^{\nu} \frac{d \rho}{1-\rho^{4}} \sqrt{1-v^{2}-\rho^{4}}\left[\left(\frac{\nu^{4}\left(1-\rho^{4}\right)}{\rho^{4}\left(1-\nu^{4}\right)} \frac{\mathrm{e}^{2 h\left(\rho^{2}-\nu^{2}\right)}}{\sin ^{2} \theta_{1}}-1\right)^{-\frac{1}{2}}+\left(\frac{\nu^{4}\left(1-\rho^{4}\right)}{\rho^{4}\left(1-\nu^{4}\right)} \frac{\mathrm{e}^{2 h\left(\rho^{2}-\nu^{2}\right)}}{\sin ^{2} \theta_{2}}-1\right)^{-\frac{1}{2}}\right],
$$

where $\nu=\frac{r_{0}}{r_{h}}$ and $\theta_{i}$ is an angular parameter corresponding to the $i$ string. Clearly, $\ell$ is a function of several variables.

The parameters $\theta_{i}$ are, however, subject to the constraints which are the gluing conditions [Eqs. (B3) and (B4)]. In the model we are considering, these conditions take the form

$$
\begin{gathered}
\sin \theta_{1}+\sin \theta_{2}+\phi=0, \\
\cos \theta_{1}+\cos \theta_{2}-\sqrt{1-\phi^{2}}-3 \kappa \sqrt{1-\nu^{4}}\left(1+4 h \nu^{2}+\frac{2 \nu^{4}}{1-v^{2}-\nu^{4}}\right) \mathrm{e}^{-3 h \nu^{2}}=0,
\end{gathered}
$$


with

$$
\phi=\frac{v \nu^{2} \mathrm{e}^{h\left(\sqrt{1-v^{2}}-\nu^{2}\right)}}{\sqrt{\left(1-v^{2}\right)\left(1-\nu^{4}\right)}} .
$$

With this at hand, $\ell$ can be viewed as a function of three variables $\nu, v$, and $h$.

The important observation for what follows is that the first string changes its shape with increase of speed. The transition from configuration I to configuration II occurs at a certain "critical" speed between slow and medium, and it has a clear geometrical interpretation. It happens when $\tan \alpha_{1}=\infty$ or, equivalently, $\cos \theta_{1}=0{ }^{6}$ Loosely speaking, the end point $V$ looks like a turning point of a function $r(\xi)$, as approached from the right. Therefore, there is no contribution from string 1 stretched between $Q_{1}$ and $V$ to the force balance along the $r$ direction. A more formal way to say this is to write Eqs. (3.5a) and (3.5b) as

$$
\begin{aligned}
& \sqrt{\phi(2-\phi)}-\sqrt{1-\phi^{2}}-3 \kappa \sqrt{1-\nu^{4}} \\
& \times\left(1+4 h \nu^{2}+\frac{2 \nu^{4}}{1-v^{2}-\nu^{4}}\right) \mathrm{e}^{-3 h \nu^{2}}=0 .
\end{aligned}
$$

A solution $v(\nu, h)$ of this equation is a "critical" speed, above which string 1 is bent into a $\mathrm{U}$ shape.

\section{Configuration II}

Similarly, for configuration II, we use Eqs. (A11) and (A25) to deduce the formula for the distance between the quarks:

$$
\begin{aligned}
\ell(\nu ; v, h)= & \sqrt{\frac{h}{\mathfrak{S}}} \int_{0}^{\nu} \frac{d \rho}{1-\rho^{4}} \sqrt{1-v^{2}-\rho^{4}}\left[\left(\frac{\nu^{4}\left(1-\rho^{4}\right)}{\rho^{4}\left(1-\nu^{4}\right)} \frac{\mathrm{e}^{2 h\left(\rho^{2}-\nu^{2}\right)}}{\sin ^{2} \theta_{1}}-1\right)^{-\frac{1}{2}}+\left(\frac{\nu^{4}\left(1-\rho^{4}\right)}{\rho^{4}\left(1-\nu^{4}\right)} \frac{\mathrm{e}^{2 h\left(\rho^{2}-\nu^{2}\right)}}{\sin ^{2} \theta_{2}}-1\right)^{-\frac{1}{2}}\right] \\
& +2 \sqrt{\frac{h}{\mathfrak{S}}} \int_{\nu}^{\lambda} \frac{d \rho}{1-\rho^{4}} \sqrt{1-v^{2}-\rho^{4}}\left(\frac{\nu^{4}\left(1-\rho^{4}\right)}{\rho^{4}\left(1-\nu^{4}\right)} \frac{\mathrm{e}^{2 h\left(\rho^{2}-\nu^{2}\right)}}{\sin ^{2} \theta_{1}}-1\right)^{-\frac{1}{2}} .
\end{aligned}
$$

Here $\lambda=\frac{r_{c}}{r_{h}}$, with $r_{c}$ being a coordinate of the turning point (see Fig. 10). Importantly, $r_{c}$ obeys the condition (A24), which now can be written as

$$
\left(1-\lambda^{-4}\right) \mathrm{e}^{2 h \lambda^{2}}=\sin ^{2} \theta_{1}\left(1-\nu^{-4}\right) \mathrm{e}^{2 h \nu^{2}} .
$$

It allows one to reduce the number of parameters describing the configuration by 1 .

We can further reduce the number of parameters by the gluing conditions. One of those is written above as Eq. (3.5a), and the other is obtained from Eq. (B5):

$$
\begin{aligned}
& \cos \theta_{1}-\cos \theta_{2}+\sqrt{1-\phi^{2}}+3 \kappa \sqrt{1-\nu^{4}} \\
& \times\left(1+4 h \nu^{2}+\frac{2 \nu^{4}}{1-v^{2}-\nu^{4}}\right) \mathrm{e}^{-3 h \nu^{2}}=0 .
\end{aligned}
$$

As in the previous subsection, it is possible, at least numerically, to solve the system of gluing equations to get a reduced description with fewer parameters. Thus, we can write $\ell$ as a function of three variables $\nu, v$, and $h$.

As speed increases, string 2 stretched between $Q_{2}$ and $V$ becomes more and more straight, until at angle $\theta_{2}=0$ the transition to configuration III occurs at a certain "critical" speed between medium and high. Setting $\theta_{2}=0$ in Eqs. (3.5a) and (3.10), we find

\footnotetext{
${ }^{6}$ The convention adopted in Appendix $\mathrm{A}$ is that $\theta_{1}$ is negative. So, this corresponds to $\sin \theta_{1}=-1$.
}

$$
\begin{aligned}
1- & 2 \sqrt{1-\phi^{2}}-3 \kappa \sqrt{1-\nu^{4}} \\
& \times\left(1+4 h \nu^{2}+\frac{2 \nu^{4}}{1-v^{2}-\nu^{4}}\right) \mathrm{e}^{-3 h \nu^{2}}=0 .
\end{aligned}
$$

A solution $v(\nu, h)$ is a "critical" speed above which the diquark configuration looks like that of Fig. 4 on the right.

It is noteworthy that at the transition from configuration II to configuration III, string 1 touches the induced horizon. This follows from Eqs. (3.5a) and (3.9). Indeed, a short calculation shows that $\lambda=\sqrt[4]{1-v^{2}}$. If so, then as explained in Appendix A, a cusp forms exactly at the touching point, and the corresponding cusp angle is

$$
\tan \gamma=\frac{1}{v}\left(\frac{1-v^{2}}{1-2 h v^{2} \sqrt{1-v^{2}}}\right)^{\frac{1}{2}}
$$

It is well defined because $h<1$.

\section{Configuration III}

Now, consider configuration III. While the two previous configurations do not involve something so unusual, this does. The first point is that a cusp appears on string 1 . In contrast to the case of motion with the "critical speed" given by Eq. (3.11), for higher speed the cusp angle is simply 


$$
\gamma=0
$$

as follows from Eq. (A32).

To obtain from the formulas (A11) and (A29) the expression for the distance between the quarks, we will have to subtract one expression from another, as is clear from Fig. 4 on the right. As a result, we find

$$
\begin{aligned}
\ell(\nu ; v, h)= & \sqrt{\frac{h}{\mathfrak{S}}} \int_{0}^{\nu} \frac{d \rho}{1-\rho^{4}} \sqrt{1-v^{2}-\rho^{4}}\left[\left(\frac{\nu^{4}\left(1-\rho^{4}\right)}{\rho^{4}\left(1-\nu^{4}\right)} \frac{\mathrm{e}^{2 h\left(\rho^{2}-\nu^{2}\right)}}{\sin ^{2} \theta_{1}}-1\right)^{-\frac{1}{2}}-\left(\frac{\nu^{4}\left(1-\rho^{4}\right)}{\rho^{4}\left(1-\nu^{4}\right)} \frac{\mathrm{e}^{2 h\left(\rho^{2}-\nu^{2}\right)}}{\sin ^{2} \theta_{2}}-1\right)^{-\frac{1}{2}}\right] \\
& +2 \sqrt{\frac{h}{\mathfrak{S}}} \int_{\nu}^{\sqrt[4]{1-v^{2}}} \frac{d \rho}{1-\rho^{4}} \sqrt{1-v^{2}-\rho^{4}}\left(\frac{\nu^{4}\left(1-\rho^{4}\right)}{\rho^{4}\left(1-\nu^{4}\right)} \frac{\mathrm{e}^{2 h\left(\rho^{2}-\nu^{2}\right)}}{\sin ^{2} \theta_{1}}-1\right)^{-\frac{1}{2}} .
\end{aligned}
$$

The second point is that there is a limiting speed, above which $\ell$ vanishes:

$$
\ell\left(\nu ; v_{\lim }, h\right)=0 .
$$

What happens is that as speed increases, quark 2 "approaches" closer and closer to quark 1 and finally "collides" with it. This intuitively obvious fact can be checked numerically using Eqs. (3.14) and (3.15). The results show that $v_{\text {lim }}$ may be well below unity. ${ }^{7}$ From this, one can draw the conclusion that the length contraction is not identical to that of special relativity. Of course, it is expected that the length contraction in the thermal medium need not be Lorentzian type, since the medium defines a preferred rest frame.

We conclude our discussion of configuration III with a remark on what needs to be said about the gluing conditions at the vertex. In fact, these conditions are similar to those of configuration II, and therefore are given by Eqs. (3.5a) and (3.10).

\section{Some analysis}

Let us first recall the description in Ref. [8] of the static diquark configuration of Fig. 2. In that analysis, we showed that in the $\overline{\mathbf{3}}$ channel there are two branches of $\ell(\nu ; h)$, and each of those is bounded from above. This means that the connected configuration exists only if $\ell$ does not exceed the critical distance. We pick up the branch of the solution which leads to $F_{\mathrm{QQ}}^{\overline{3}} \sim-\frac{1-m_{\mathrm{D}} \ell}{\ell}$ as $\ell \rightarrow 0$, with $m_{\mathrm{D}}$ being the Debye mass. In this case, the corresponding formulas can be obtained from those of Sec. III B 1 by setting $v=0$. The parameter $\nu$ takes values in the interval $\left[0, \nu_{*}\right]$. The function $\ell(\nu ; h)$ grows with $\nu$ so that $\ell\left(\nu_{*} ; h\right)$ is the critical distance at given temperature. Note that $\nu_{*}$ is determined by temperature.

In addition, let us make a simple estimate of the dissociation temperature based on $\xi_{\mathrm{s}}\left(T_{\text {diss }}\right) \sim 2 \varrho$, where

\footnotetext{
${ }^{7}$ See also Fig. 5 on the left.
}

$\xi_{\mathrm{s}}$ is a screening length and $\varrho$ a diquark radius at zero temperature. Treating the screening length as the Debye length $\xi_{\mathrm{s}}=1 / m_{D}$ and using the expression for the Debye mass [8]

$$
\frac{m_{\mathrm{D}}}{T}=a-b \frac{T_{c}^{2}}{T^{2}}
$$

we get

$$
T_{\mathrm{diss}} \sim \frac{1}{4 a \varrho}\left(1+\sqrt{1+16 a b \varrho^{2} T_{c}^{2}}\right) .
$$

For $S U(3)$ pure gauge theory with $T_{c}=260 \mathrm{MeV}, a=$ 0.71 and $b=0.24$. This yields $T_{\text {diss }} \sim 2.1 T_{c}(c c)$ and $T_{\text {diss }} \sim 2.9 T_{c}(b b)$, with the radii $0.28 \mathrm{fm}(c c)$ and $0.19 \mathrm{fm}(b b)$ obtained from an effective quark-quark potential of the "Cornell" Coulomb + linear form at half strength [2]. As expected, the bottomonium diquarks dissociate at higher temperatures. These simple estimates are quite similar to those based on the analysis of the Schrödinger equation for diquark states in two-flavor QCD [3].

Now, suppose that the static configuration is boosted so that it moves along the quark-quark axis. In this situation (at late times), there are three possible shapes of the configuration, as shown in Fig. 4. Each can be described by the three parameters $\nu, v$, and $h$. In general, the parameter space of the model is quite complicated, but there exist some relatively simple divisions. For example, when the temperature range is narrow and restricted to a region above $T_{c}$, say $T_{c} \lesssim T \lesssim 3 T_{c}$, there are no singularities. $^{8}$ This range is of great interest, as the dual gauge theory is so strongly coupled that it makes its study difficult. In Fig. 5 on the left, we present a two-dimensional slice of the parametric space which contains three regions according to a number of the string configurations of

\footnotetext{
${ }^{8}$ Note that it corresponds to $\frac{1}{9} \lesssim h \lesssim 1$.
} 

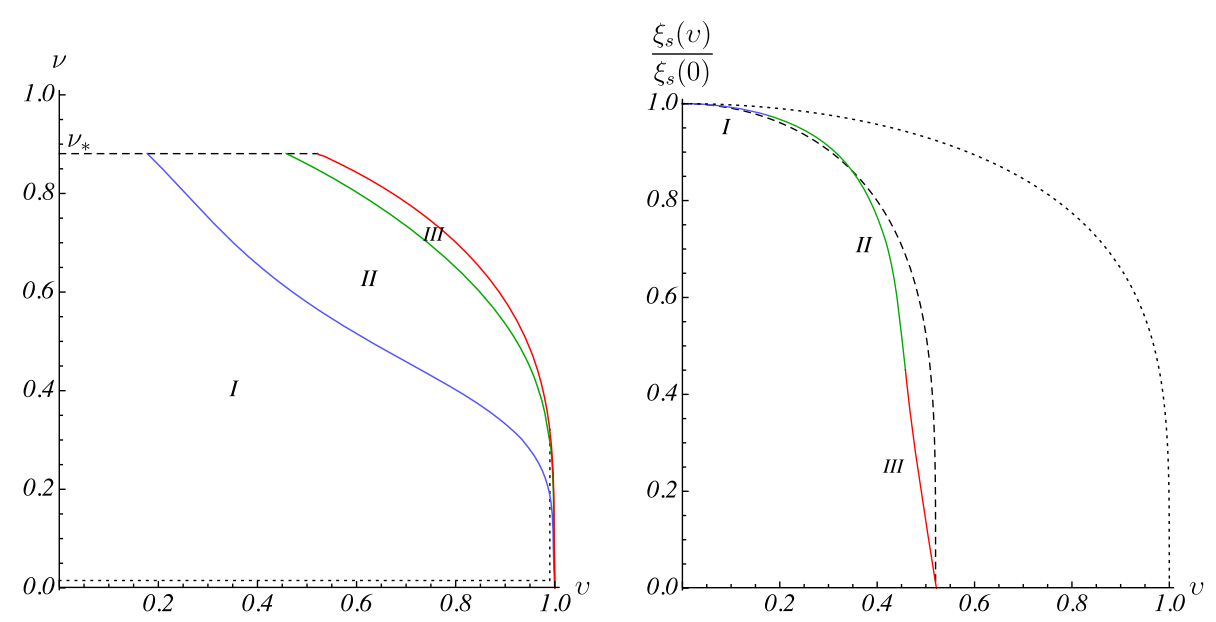

FIG. 5. The parametric space at $h=\frac{4}{9}$, and thus $\nu_{*} \approx 0.88$. The labeling is according to Fig. 4. (Left) A temperature slice of the parametric space. The dashed line represents the maximal value of $\nu$. The dotted lines indicate cutoffs. The colored curves from left to right correspond to Eqs. (3.7), (3.11), and (3.15). (Right) $\xi_{s}=\ell\left(\nu_{*} ; v, h\right)$ as a function of $v$. Here $v_{\text {lim }} \approx 0.52$. Each of the different colored parts of the curve is plotted by using the corresponding formula for $\ell$. The dotted and dashed curves are defined by Eqs. (3.18) and (3.19).

Secs. III B 1-3. It is actually nothing special, but a couple of points are worthy of note.

From the gluing conditions (3.5b) and (3.10), it follows that there are no solutions with $\nu=\sqrt[4]{1-v^{2}}$. Such a curve always lies outside the allowed region of the parametric space, and therefore is not shown in the figure. In other words, the baryon vertex never touches the induced horizon. It is always located between the induced horizon and boundary. This implies that the right-hand side of Eq. (2.8) is real, and therefore, the tachyonic instability does not occur.

The effective string model we are considering here suffers from power divergences and requires a suitable regularization. These divergences are usually associated with infinitely heavy quark sources placed on the boundary. One way to deal with this situation is to impose a lower bound (cutoff) $\varepsilon$ on the radial coordinate $r$. If so, then $\nu$ is also bounded from below by $\frac{\varepsilon}{r_{h}}$. The corresponding cutoff is indicated by the dotted horizontal line. But this is not the whole story for the diquark configuration. The point is that the induced horizon approaches the boundary as speed increases. Clearly, the string construction make sense only when the induced horizon is above the cutoff, namely $r_{v}>\varepsilon$. This leads to a upper bound on $v$. In particular, with Eq. (3.2), it is given by $v<\sqrt{1-\frac{\varepsilon^{4}}{r_{h}^{4}}}$ This is indicated by the vertical dotted line. Since the calculation of the drag force does not suffer from the problem of infinities, there is no need for us to go further into this aspect.

The function $\ell(\nu ; v, h)$ is rather complicated and defined piecewise by the corresponding integral expression in each region of the parameter space. We will now analyze it for large and small $\nu$. Both are of special interest for phenomenology.
We begin with large $\nu$. The alternative to the Debye screening length defined directly from the correlator of Polyakov loops is to define the screening length as $\xi_{\mathrm{s}} \equiv$ $\ell\left(\nu_{*} ; v, h\right)$ [15]. ${ }^{9}$ For a quark-antiquark pair, such a definition yields a peculiar form of length contraction:

$$
\xi_{\mathrm{s}}(v) \approx \sqrt[4]{1-v^{2}} \xi_{\mathrm{s}}(0)
$$

In Fig. 5 on the right, we plot $\xi_{s}$ given piecewise by the formulas (3.4), (3.8), and (3.14) at $\nu=\nu_{*}$. Obviously, it is much different from what is expected from Eq. (3.18). The reason is simple and straightforward: the existence of a limiting speed. It thus makes sense to take this fact into account and consider a modification of (3.18):

$$
\xi_{\mathrm{s}}(v) \approx \sqrt[4]{1-\frac{v^{2}}{v_{\mathrm{lim}}^{2}}} \xi_{\mathrm{s}}(0) .
$$

As seen from the figure, this is a quite acceptable approximation, especially when the speed is slower than 0.45 .

Next, let us consider what happens when $\nu$ is small. It follows from the structure of the parametric space, and in particular from the left panel of Fig. 5, that in this case only region I matters, so that $v_{\lim } \rightarrow 1$ as $\nu \rightarrow 0$. The analysis of Eq. (3.4) for small $\nu$ is simple, and the first two Taylor coefficients are computed in Appendix C. So, one is led to suspect that in this limit,

\footnotetext{
${ }^{9}$ Even in the anti-de Sitter (AdS) case, this definition requires a caveat. The problem is incompleteness at larger $\nu$. One way to address this problem is to introduce one more diagram as suggested in Ref. [16]. However, it remains to be seen whether both definitions of the screening length will agree.
} 


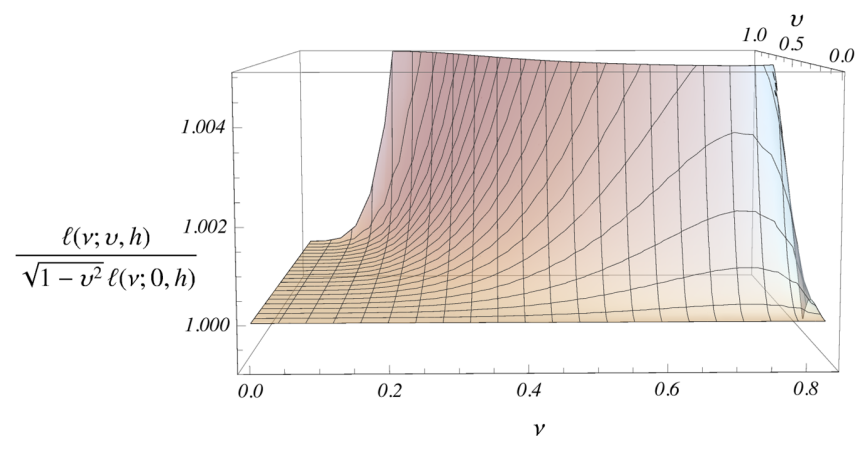

FIG. 6. $\ell(\nu ; v, h) / \sqrt{1-v^{2}} \ell(\nu ; 0, h)$ as a function of $\nu$ and $v$ at $h=\frac{4}{9}$.

$$
\ell(\nu ; v, h) \approx \sqrt{1-v^{2}} \ell(\nu ; 0, h)
$$

which is nothing else but the Lorentz contraction formula. In Fig. 6 , we plot the ratio $\ell(\nu ; v, h) / \sqrt{1-v^{2}} \ell(\nu ; 0, h)$ at $h=\frac{4}{9}$, which enables us to gain more detailed insight into the approximation (3.20). The plot is restricted to the range 1 to 1.005 . We see that the $v$ dependence is in fact of Lorentzian form up to $\nu=0.15$, but for larger values it gets more involved. Heuristically, we can say that the formula (3.20) holds as long as the vertex remains much closer to the boundary than to the black hole horizon.

Now a question arises: What approximation might be relevant to the Debye screening length, as considered in Ref. [8]? No real answer will be given here, but we plan to address this issue in future research.

\section{CONCLUDING COMMENTS}

(i) The purpose of this paper has been to initiate discussions on heavy diquarks as probes of strongly coupled plasma. As the first step in this direction, we considered the drag force on a test diquark by using the five-dimensional effective string models. Certainly, there are many topics and issues which deserve to be studied in depth and with the greatest seriousness. Some of those are mentioned in Ref. [5], although in the context of heavy quarks-in particular, momentum broadening and disturbance of the plasma induced by test probes.

(ii) The nonrelativistic limit of Eq. (2.15) is that the frag force is proportional to the spatial string tension. ${ }^{10}$ We have [7]

$$
F_{\text {drag }}=-\sigma_{s} v,
$$

with $\sigma_{s}=\mathfrak{g} w\left(r_{h}\right)$ being the spatial string tension. This follows from the fact that $r_{v} \rightarrow r_{h}$ as $v \rightarrow 0$.

\footnotetext{
${ }^{10}$ In the AdS case, this is always true, no matter what speed a single quark (diquark) is moving [17].
}

Thus, when the motion is nonrelativistic, the spatial string tension plays a pivotal role in controlling how strong the drag force is. Using the models at our disposal, it is possible to make some estimates. In Ref. [7], we got a couple of estimates at zero baryon chemical potential $\mu$. The goal here is to make a simple estimate of $\sigma_{s}$ at nonzero chemical potential. To this end, we follow the lines of Ref. [8] and involve the one-parameter deformation of the Reissner-Nordström-AdS black hole solution [18], with the warp factor $w(r)=\frac{\mathrm{e}^{\xi r^{2}}}{r^{2}}$, where $\mathfrak{g}$ is a deformation parameter. ${ }^{11}$ As explained in Appendix C of Ref. [8], one can think of $T$ and $\mu$ as functions of two parameters:

$$
\begin{aligned}
& T(h, q)=\frac{9}{8 \pi} \frac{\sqrt{\mathfrak{g}}\left(1-q^{2}\right) h^{\frac{3}{2}}}{\mathrm{e}^{\frac{3}{2} h}-1-\frac{3}{2} h}, \\
& \mu(h, q)=\frac{2 \sqrt{3 \mathfrak{s}} \mathrm{r} q\left(1-\mathrm{e}^{-\frac{1}{2} h}\right)}{\left(9+(7+6 h) \mathrm{e}^{-2 h}-16 \mathrm{e}^{-\frac{1}{2} h}\right)^{\frac{1}{2}}} .
\end{aligned}
$$

Here, as before, $h=\mathfrak{s} r_{h}^{2}$, while $q$ and $\mathfrak{r}$ are new parameters. The parameter $q$ is related to a black hole charge. It takes values in the interval $[0,1]$. The parameter $\mathfrak{r}$ is considered as free.

Given the formal formulas that we have described above, it seems straightforward to apply those and determine the spatial string tension as a function of $T$ and $\mu$. In the left panel of Fig. 7 we plot this function, normalized by the string tension $\sigma$ at zero temperature and chemical potential. ${ }^{12}$ Units are set by $T_{p c}=0.26 \sqrt{\mathfrak{g}}$ and $\mu_{p c}=2 \sqrt{\mathfrak{g}}$, as in Ref. [8] for two-flavor QCD [19]. We see that $\sigma_{s}$ is increasing with $T$, and more slowly with $\mu$. Additionally, we consider the Taylor expansion of $\sigma_{s}(T, \mu)$ about the point $(T, 0)$. In the model under consideration, it is given by

$$
\sigma_{s}(T, \mu)=\sum_{n=0}^{\infty} \sigma_{s}^{(2 n)}\left(\frac{\mu}{T}\right)^{2 n}
$$

The coefficients $\sigma_{s}^{(2 n)}$ can be derived directly from Eqs. (4.1) and (4.2). The first two are presented in Appendix D. Note that in this expansion, odd powers of $\mu$ do not appear, because $h$ is an even function of $\mu$. Since small $\mu$ is of great interest for lattice QCD, in the right panel of Fig. 7 we present the result for the ratio $\frac{\sigma_{s}^{(2)}}{\sigma_{s}^{(0)}}$.

\footnotetext{
${ }^{11}$ In Ref. [8], this model was used to estimate the Debye screening masses near the critical line in the $\mu-T$ plane of twoflavor QCD [19]. Now we wish to supplement our list with the estimate of the spatial string tension.

${ }^{12}$ Such a tension is simply $\sigma=\mathfrak{g} \mathfrak{g e}$.
} 

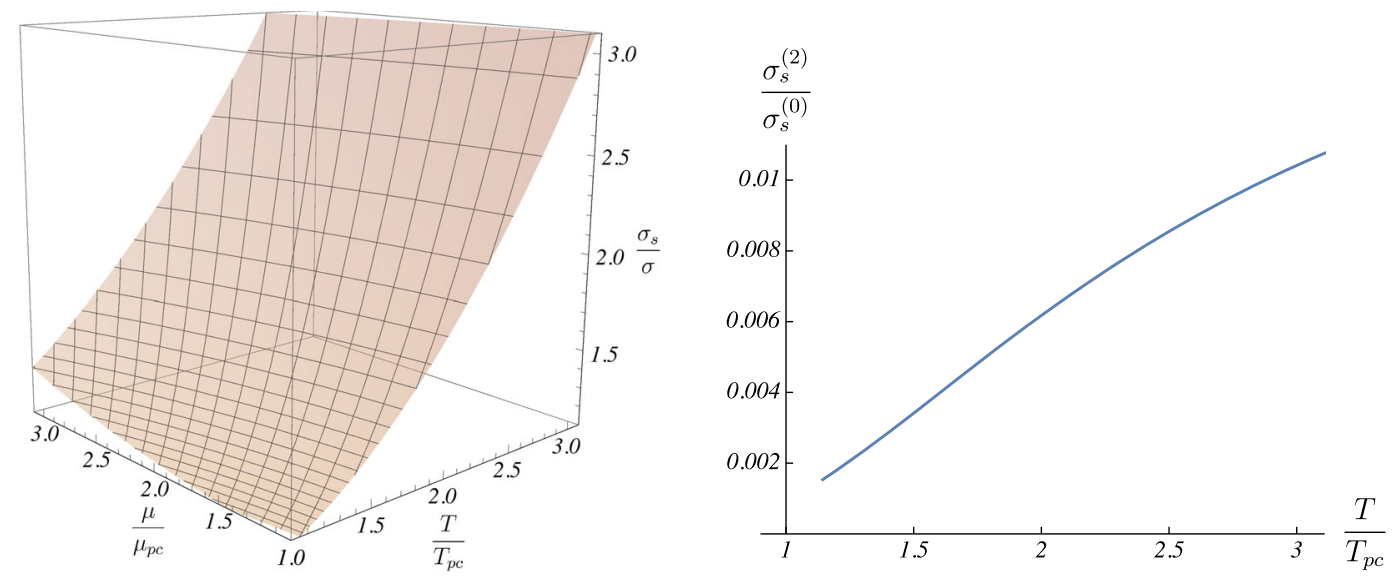

FIG. 7. Left: The ratio $\frac{\sigma_{s}}{\sigma}$ versus temperature and baryon chemical potential. Here $\mathfrak{r}=6$. Right: The ratio $\frac{\sigma_{s}^{(2)}}{\sigma_{s}^{(0)}}$ versus temperature.
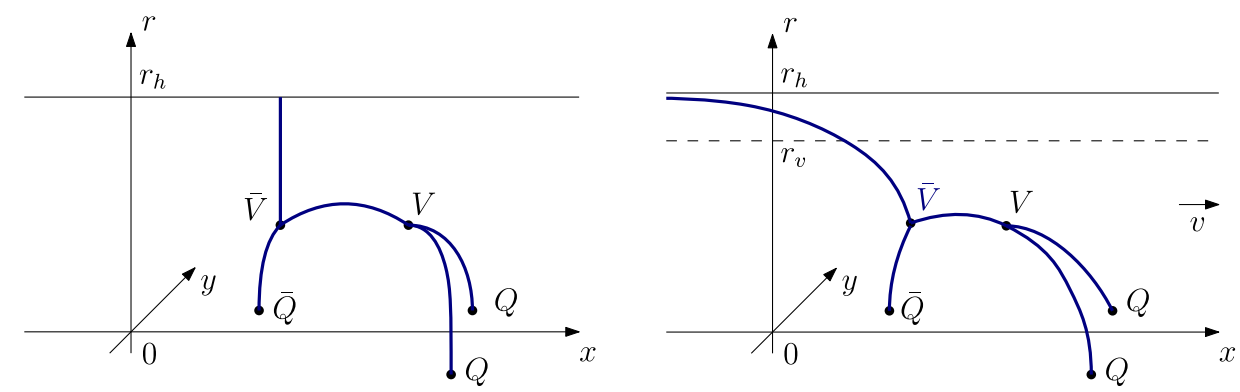

FIG. 8. String configurations of heavy triquarks in the color $\mathbf{3}$ channel. The heavy quarks $Q$ and antiquark $\bar{Q}$ are placed on the boundary of space. The strings are stretched between the quark sources, vertices, and horizon. (Left) A static configuration. (Right) A configuration moving with speed $v$ in the $x$ direction.

Both results are predictions, as so far there are no lattice studies available. However, this requires a caveat: in contrast to lattice QCD, the model we are considering has no explicit dependence on quark masses.

(iii) Another interesting concept is that of a triquark. It naturally occurs in hadron spectroscopy-for example, by treating pentaquark states as bound states of color antitriplet diquarks and color triplet triquarks. ${ }^{13}$

In the static case, it is straightforward to build a string configuration for a triply heavy triquark in the deconfined phase, as is shown in the left panel of Fig. 8. What distinguishes it from the previous configurations is that it contains an antibaryon vertex $\bar{V}$-that is, it is a vertex connecting three antiquarks to form an antibaryon. As in Sec. III, we should now ask what is the drag force acting on a triquark moving in a hot medium. To this end, we consider a triquark configuration moving with speed $v$, as sketched in the right panel of Fig. 8. If we proceed in this way, arguments similar to those of Sec. III show that the drag force is given by Eq. (2.15). In other words, if

\footnotetext{
${ }^{13}$ For some examples, see Ref. [20].
}

the quark's relative motion is negligible, then the drag force is independent of a number of constituents. What matters, however, is a number of trailing strings (representation of color group). In all the cases, there is a single trailing string that corresponds to the color triplet-antitriplet channel.

It is worthy of noting that there is one aspect to a triquark string configuration which is peculiar to the case of any multiquark configuration, and that is its instability due to $V \bar{V}$ annihilation. One example of this is a well-known flip-flop for the tetraquark potential. This has a natural interpretation in string theory [21]. The point is that $V \bar{V}$ is nothing else but a brane-antibrane system. For such a system, there is a critical separation such that a tachyonic mode develops for smaller separations. The tachyonic instability represents a flow toward annihilation of the brane-antibrane system. If it occurs in a triquark configuration, the configuration decays to two configurations, one for a single quark and one for a meson. We will not pursue this circle of ideas any further here and leave them for future study.

(iv) By now, there is reasonably strong evidence, mainly from lattice QCD, that the $S U(3)$ theory of quarks 
and gluons has a dual description in terms of quantum strings. Since the precise formulation of the latter is still not known, what we can do is to gain useful insight and grow with each success of the effective string model already at our disposal. One might think of criticizing this model on several grounds, in particular because of the use of string theory to describe a gauge theory with a finite number of colors. This is, in fact, related to the long-standing question, namely whether $N_{c}=3$ is good enough for the $1 / N_{c}$ expansion in QCD. We have nothing to say at this point, except that we hope to return to this issue in future work.

\section{ACKNOWLEDGMENTS}

We would like to thank I. Aref'eva, P. de Forcrand, S. Hofmann, R. Metsaev, P. Weisz, and U. A. Wiedemann for useful and encouraging discussions. We also thank the Arnold Sommerfeld Center for Theoretical Physics and CERN Theory Division for the warm hospitality. This work was supported in part by Russian Science Foundation Grant No. 16-12-10151.

\section{APPENDIX A: A NAMBU-GOTO STRING MOVING WITH CONSTANT SPEED}

Here we explore in some detail a few examples of strings steadily moving in the five-dimensional spacetime whose metric is given by Eq. (2.1). This provides the basis for the practical calculations of Sec. III. We consider the case where the horizon is closer to the boundary than the socalled soft wall. This guarantees that a dual gauge theory is deconfined [12].

The motion of strings is studied using the Nambu-Goto action

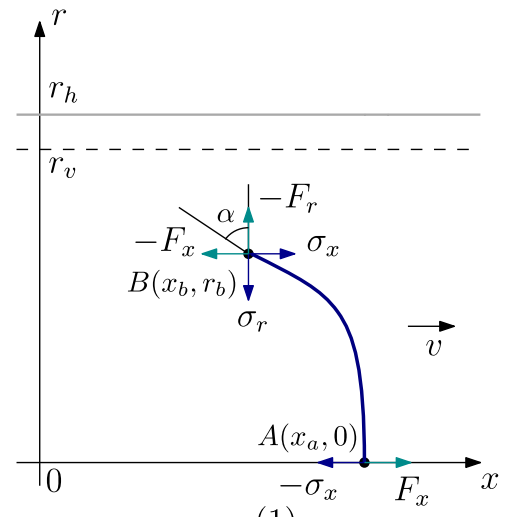

(1)

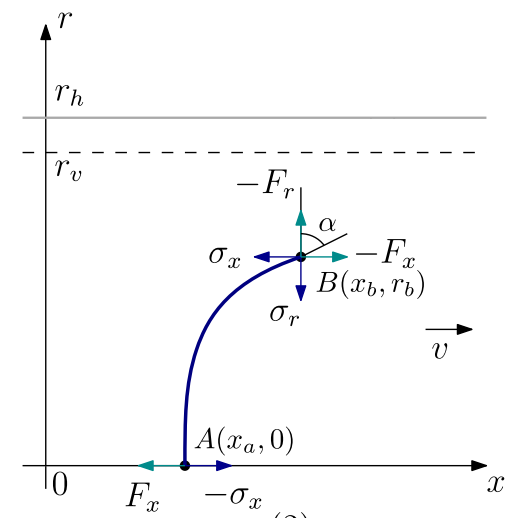

(2)

$$
S=-\frac{1}{2 \pi \alpha^{\prime}} \int_{0}^{1} d \sigma \int d \tau \sqrt{-\gamma}, \quad \gamma=\operatorname{det} \gamma_{\alpha \beta},
$$

where $\alpha^{\prime}$ is a string constant, $(\tau, \sigma)$ are world-sheet coordinates, and $\gamma_{\alpha \beta}$ is an induced metric.

\section{Single-string configurations}

We begin with what are perhaps the elementary examples. These are the cases, denoted in Fig. 9 as configurations 1 and 2, in which one string end point is on the boundary at $r=0$, while another in the bulk at $r=r_{b}$. In each case, the string lies on the $x$ - $r$ plane and uniformly moves in the $x$ direction with speed $v$. We consider a lab frame which is at rest and related to the thermal medium of a dual gauge theory.

Since we are interested in uniform motion, we choose the static gauge $t=\tau$ and $r(\sigma)=c_{1} \sigma+c_{0}$. Combining the latter with the boundary conditions at the end points

$$
r(0)=0, \quad r(1)=r_{b},
$$

we obtain $c_{1}=r_{b}$ and $c_{0}=0$. For $x(t, r)$, we take the ansatz of Ref. [6]

$$
x(t, r)=v t+\xi(r) .
$$

In light of the applications of string theory to heavy ion collisions, it describes the late-time behavior of strings attached to heavy quark sources on the boundary.

Having taken the ansatz, we can write down the induced metric in a form suitable for our further purposes. It is simply

$$
\begin{aligned}
d s^{2}= & w R^{2}\left(-\left(f-v^{2}\right) d t^{2}+2 v \partial_{r} \xi d t d r\right. \\
& \left.+\left(f^{-1}+\left(\partial_{r} \xi\right)^{2}\right) d r^{2}\right),
\end{aligned}
$$

FIG. 9. Single-string configurations at time $t=0$. In all the cases, the string moves with speed $v$ in the $x$ direction. $r_{h}$ and $r_{v}$ are the horizon locations of the spacetime and induced metrics, respectively. The $F_{i}$ 's stand for some external forces exerted on the string end points to balance internal string tensions $\sigma_{i}$ and keep the string in uniform motion. In configurations 1 and 2 , the string is of finite length and is stretched between the points $A$ and $B$. In configuration 3, the string is of infinite length and is trailing out behind the end point $B$. 
where $\partial_{r} \xi$ means $\frac{\partial \xi}{\partial r}$. Importantly, the induced metric has a horizon at $r=r_{v}$, because the equation $f(r)=v^{2}$ has a solution on the interval $\left[0, r_{h}\right]$. We call it an induced horizon. ${ }^{14}$ The Nambu-Goto action now takes the form

$$
S=-\mathfrak{g} \int d t \int_{0}^{r_{b}} d r w \sqrt{1-\frac{v^{2}}{f}+f\left(\partial_{r} \xi\right)^{2}},
$$

with $\mathfrak{g}=\frac{R^{2}}{2 \pi \alpha^{\prime}}$.

In the presence of external forces exerted on the string end points, the total action $\mathcal{S}$ includes, in addition to the standard Nambu-Goto action, boundary terms arising from the coupling with external fields. For the purposes of this paper, we do not need to make them explicit. What we do need is a variation of $\mathcal{S}$ with respect to the field $\xi$ as well as with respect to $x_{a}, x_{b}$, and $r_{b}$ describing the location of the string end points. Since the motion is uniform, we can choose $t=0$, as illustrated in Fig. 9. Then $\delta \xi(0)=\delta x_{a}$ and $\delta \xi\left(r_{b}\right)=\delta x_{b}-\partial_{r} \xi \delta r_{b} .{ }^{15}$ After a simple calculation, we find ${ }^{16}$

$$
\begin{aligned}
\delta \mathcal{S}= & -\int d t \int_{0}^{r_{b}} d r \partial_{r} \sigma_{x} \delta \xi+\int d t\left(\sigma_{x}-F_{x}\right)\left(\delta x_{b}-\delta x_{a}\right) \\
& +\left(\sigma_{r}-F_{r}\right) \delta r_{b},
\end{aligned}
$$

with

$$
\sigma_{x}=-\frac{\mathfrak{g} w f \partial_{r} \xi}{\sqrt{1-\frac{v^{2}}{f}+f\left(\partial_{r} \xi\right)^{2}}}, \quad \sigma_{r}=-\frac{\mathfrak{g} w\left(1-\frac{v^{2}}{f}\right)}{\sqrt{1-\frac{v^{2}}{f}+f\left(\partial_{r} \xi\right)^{2}}}
$$

Here we assume that the end point $A$ is fixed in the $r$ direction, and as a consequence of that, $\delta r_{a}=0$.

A pair of comments about Eqs. (A6) and (A7) is in order. First, $\sigma_{x}=c$ is the first integral of the equation of motion for $\xi$, and therefore an absolute value of $\sigma_{x}$ must be the same at both end points. The force balance is the reason why $F_{x}$ must also obey this requirement. All together, this determines the form of the boundary terms in Eq. (A6). Second, the $\sigma_{i}$ 's are nothing else but the components of the world-sheet current $\mathcal{P}_{\mu}^{\sigma}$ [22]. Explicitly, $\sigma_{x}=\mathcal{P}_{x}^{\sigma}=\frac{\delta S}{\delta \xi^{\prime}}$ and $\sigma_{r}=\mathcal{P}_{r}^{\sigma}=\frac{\delta S}{\delta r^{\prime}}$, where a prime denotes a derivative with respect to the world-sheet coordinate $\sigma$.

For what follows, it is convenient to express the integration constant $c$ in terms of $r_{b}$ and $\tan \alpha=\left.\partial_{r} \xi\right|_{r_{b}}$, and to perform the following rescaling:

\footnotetext{
${ }^{14}$ This naming convention is shorthand for saying that it is related to the induced metric on the world sheet.

${ }^{15}$ The latter follows from the chain rule because the field $\xi$ depends on $r_{b}$ according to Eq. (A2).

${ }^{16}$ This equation also defines our sign conventions for the $\sigma_{i}$ 's and $F_{i}$ 's.
}

$$
\tan \alpha=\frac{\sqrt{1-\frac{v^{2}}{f}}}{\sqrt{f}} \tan \theta .
$$

At the point $B$, the $\sigma$ 's are then

$$
\sigma_{x}=-\bar{\sigma} \sin \theta, \quad \sigma_{r}=-\mathfrak{g} w \sqrt{1-\frac{v^{2}}{f}} \cos \theta,
$$

where $\bar{\sigma}=\mathfrak{g} w \sqrt{f}$. Note that $\sigma_{x}>0$ for configuration 1 , and $\sigma_{x}<0$ for configuration 2 .

The string length along the $x$ axis can be found by further integrating the first integral. First, we get from Eq. (A7)

$$
\partial_{r} \xi= \pm \frac{1}{\sqrt{f}} \sqrt{\frac{1-\frac{v^{2}}{f}}{\frac{\bar{\sigma}^{2}}{\sigma_{x}^{2}}-1}}
$$

with the minus sign for configuration 1 and the plus sign for 2. Then the integration over $r$ yields

$$
\begin{aligned}
\ell= & \left|x_{a}-x_{b}\right|=r_{h} \int_{0}^{\nu} \frac{d \rho}{f\left(r_{h} \rho\right)} \sqrt{f\left(r_{h} \rho\right)-v^{2}} \\
& \times\left(\frac{1}{\sin ^{2} \theta} \frac{\bar{\sigma}^{2}\left(r_{h} \rho\right)}{\bar{\sigma}^{2}\left(r_{h} \nu\right)}-1\right)^{-\frac{1}{2}},
\end{aligned}
$$

where $\nu=\frac{r_{b}}{r_{h}}$. In this derivation we set $t=0$. It is clear that a similar derivation for $t \neq 0$ would give the same result.

We conclude our discussion of configurations 1 and 2 with the question of mechanical equilibrium at the end points. Clearly, a net force must vanish at each end point, which, translated into mathematical language, means precisely that the boundary terms vanish in Eq. (A6). Thus, we have

$$
F_{x}-\sigma_{x}=0
$$

at the point $A$ and

$$
\sigma_{x}-F_{x}=0, \quad \sigma_{r}-F_{r}=0
$$

at the point $B$.

Now, we will briefly describe the remaining configuration of Fig. 9, which is a semi-infinite string trailing out behind the point $B$. It is an extension of what was proposed for the AdS geometry to that of Eq. (2.1). So, most of the material comes from Ref. [6].

As before, we choose the static gauge $t=\tau$ and $r(\sigma)=c_{1} \sigma+c_{0}$, which, when combined with the boundary conditions

$$
r(0)=r_{b}, \quad r(1)=r_{h},
$$

yields $c_{0}=r_{b}$ and $c_{1}=r_{h}-r_{b}$. For $x(t, r)$, we take the original ansatz (A3). Then the Nambu-Goto action becomes 


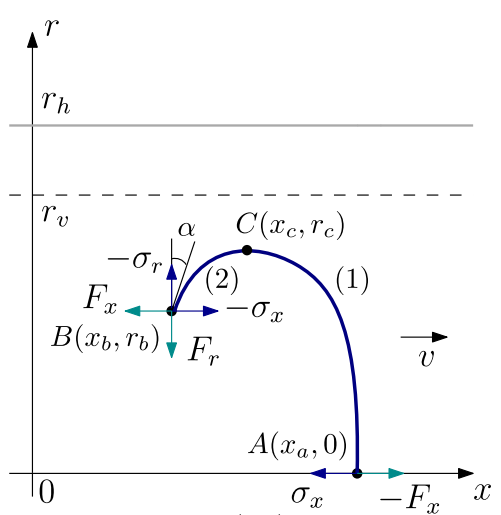

(4a)

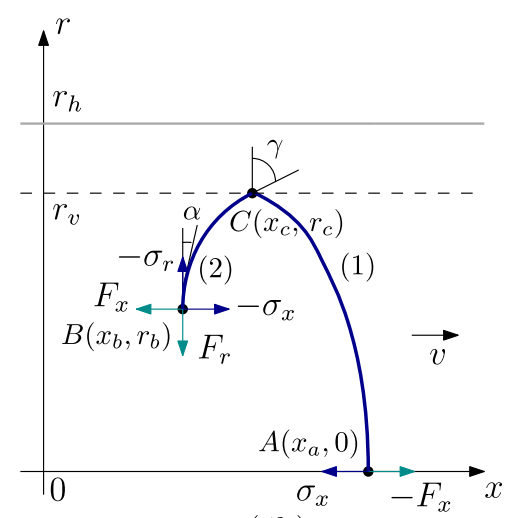

$(4 \mathrm{~b})$

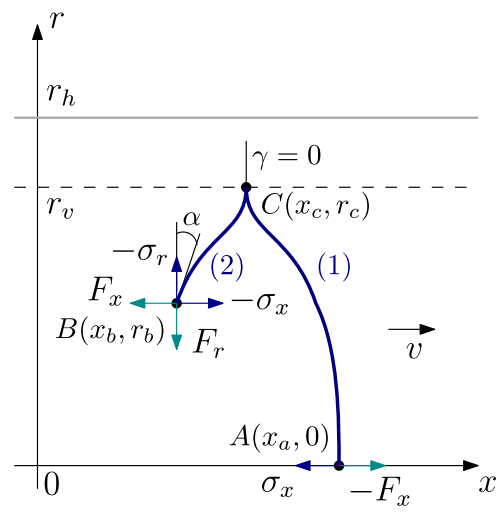

(4c)

FIG. 10. Double-string configurations at time $t=0$. In all the cases, the string moves with speed $v$ in the $x$ direction. The $F_{i}$ 's stand for some external forces to balance the internal string tensions $\sigma_{i}$. In configurations $4 \mathrm{~b}$ and $4 \mathrm{c}$, a cusp of angle $\gamma$ forms at the point $C$ lying on the induced horizon.

$$
S=-\mathfrak{g} \int d t \int_{r_{b}}^{r_{h}} d r w \sqrt{1-\frac{v^{2}}{f}+f\left(\partial_{r} \xi\right)^{2}}
$$

In the presence of external forces exerted on the end point $B$, it should be modified to include boundary terms arising from the coupling with external fields. Again, we do not need to make them explicit. We need to know the variation of the total action with respect to the field $\xi$ and coordinates of $B$. Since the motion is uniform, we consider it at time $t=0$. Then as before, a little calculation shows that the variation of $\mathcal{S}$ is given by

$$
\begin{aligned}
\delta \mathcal{S}= & -\int d t \int_{r_{b}}^{r_{h}} d r \partial_{r} \sigma_{x} \delta \xi+\int d t\left(F_{x}-\sigma_{x}\right) \delta x_{b} \\
& +\left(F_{r}-\sigma_{r}\right) \delta r_{b},
\end{aligned}
$$

where $\sigma_{x}$ and $\sigma_{r}$ are given by Eq. (A7).

As one sees from the equation of motion, $\sigma_{x}=c$ is the first integral. The point is that the only way which allows one to avoid an imaginary right-hand side in Eq. (A10) is to fix the integration constant at $r=r_{v}$. It is thus

$$
\sigma_{x}=\bar{\sigma}_{v}
$$

with $\bar{\sigma}_{v}=\bar{\sigma}\left(r_{v}\right)$. From this, at the point $B$, one deduces

$$
\sigma_{r}=-\mathfrak{g} w \sqrt{\left(1-\frac{v^{2}}{f}\right)\left(1-\frac{\bar{\sigma}_{v}^{2}}{\bar{\sigma}^{2}}\right)} .
$$

Note that, when written in this form, there is no manifest dependence on $\theta$.

Finally, we come to the question of mechanical equilibrium at the end point. The conditions for equilibrium can be read from the boundary terms in Eq. (A16). We have

$$
F_{x}-\sigma_{x}=0, \quad F_{r}-\sigma_{r}=0 .
$$

\section{2. "Double-string" configurations}

Having considered the configurations of Fig. 9, we can now complete the description of our basis. To this end, we consider the configurations of Fig. 10. There are two novelties involved. First, the approach we have used so far is now somewhat difficult to implement. The problem is that $\xi$ is a double-valued function of $r$. A possible way out, which requires a little effort, is to think off a bending string as being glued from two elementary ones. ${ }^{17}$ Obviously, each string shown in Fig. 10 may be built by gluing together the end points of the two elementary strings 1 and 2 at the turning point $C$. Second, a cusp occurs on the binding string precisely at the point at which the string touches the induced horizon. The point is that if one considers a string bit near the point $C$, its speed approaches the local speed of light as the bit approaches the induced horizon. If so, the length of the bit in the lab frame contracts towards zero. In fact, this effect is general and not restricted to either the AdS geometry ${ }^{18}$ or its generalization given by Eq. (2.1). The main reason is the induced horizon.

Before we start, let us briefly explain the appearance of these three configurations. Consider string 2 , which is stretched between the points $B$ and $C$. It is straightforward to repeat the previous analysis to show that the equation of motion can be integrated and then written as Eq. (A10). If $\frac{\bar{\sigma}^{2}}{\bar{\sigma}_{x}^{2}}-1$ has a simple zero at $r=r_{\bar{\sigma}}$ on the interval $\left[0, r_{h}\right]$, then there are three options: $r_{\bar{\sigma}}<r_{v}, r_{\bar{\sigma}}=r_{v}$, and $r_{\bar{\sigma}}>r_{v}$. These precisely correspond to the configurations $4 \mathrm{a}, 4 \mathrm{~b}$, and $4 \mathrm{c}$, shown in Fig. 10.

\footnotetext{
${ }^{17}$ This is the reason why we call the configurations of Fig. 10 "double-string" configurations.

${ }^{18}$ The occurrence of cusps on strings moving in $\mathrm{AdS}_{5}$ is known in the literature [23].
} 
We begin with configuration 4a. For the elementary strings 1 and 2, the variations of the corresponding actions can be read from Eq. (A6). Dropping all but the boundary terms at the point $C$, we have

$$
\delta S^{(i)}=\int d t \sigma_{x}^{(i)} \delta x_{c}+\sigma_{r}^{(i)} \delta r_{c}+\cdots,
$$

with $i=1,2$. Gluing the strings together requires that in the total action the boundary contributions cancel each other. So,

$$
\sigma_{x}^{(1)}+\sigma_{x}^{(2)}=0, \quad \sigma_{r}^{(1)}+\sigma_{r}^{(2)}=0 .
$$

It is easy to see that these conditions are met at the turning point $C$, at which $r_{c}=r_{\bar{\sigma}}$ holds. Indeed, we have $\sigma_{x}^{(i)}=$ $\pm \bar{\sigma}\left(r_{c}\right)$ and $\sigma_{r}^{(i)}=0$, since $\left.\partial_{r} \xi^{(i)}\right|_{r_{c}}=\mp \infty .{ }^{19}$ The latter follows directly from Eq. (A10).

Now, consider string 2. It will be useful to write the $\sigma$ 's at the point $B$ as

$$
\sigma_{x}=\bar{\sigma} \sin \theta, \quad \sigma_{r}=-\mathfrak{g} w \sqrt{1-\frac{v^{2}}{f}} \cos \theta,
$$

where we have rescaled $\tan \alpha=\left.\partial_{r} \xi\right|_{r=r_{b}}$ so that

$$
\tan \alpha=-\frac{\sqrt{1-\frac{v^{2}}{f}}}{\sqrt{f}} \tan \theta .
$$

The odd-looking minus sign comes from keeping $\theta<0$ as it is for configuration 1 of Fig. 9. Another useful relation is

$$
\bar{\sigma}\left(r_{c}\right)=-\bar{\sigma} \sin \theta .
$$

It follows from the first integral of the equation of motion and relates $r_{c}$ to $r_{b}$ and $\theta$.

Given the expression (A11), one can evaluate the string length along the $x$ direction, which is $\ell=\left|x_{a}-x_{b}\right|$, using the data at $B$ and gluing condition at $C$, with the result

$$
\begin{aligned}
\ell= & r_{h} \int_{0}^{\lambda} \frac{d \rho}{f\left(r_{h} \rho\right)} \sqrt{f\left(r_{h} \rho\right)-v^{2}}\left(\frac{1}{\sin ^{2} \theta} \frac{\bar{\sigma}^{2}\left(r_{h} \rho\right)}{\bar{\sigma}^{2}\left(r_{h} \nu\right)}-1\right)^{-\frac{1}{2}} \\
& +r_{h} \int_{\nu}^{\lambda} \frac{d \rho}{f\left(r_{h} \rho\right)} \sqrt{f\left(r_{h} \rho\right)-v^{2}}\left(\frac{1}{\sin ^{2} \theta} \frac{\bar{\sigma}^{2}\left(r_{h} \rho\right)}{\bar{\sigma}^{2}\left(r_{h} \nu\right)}-1\right)^{-\frac{1}{2}} .
\end{aligned}
$$

Here $\nu=\frac{r_{b}}{r_{h}}$ and $\lambda=\frac{r_{c}}{r_{h}}$.

If one or more external forces are exerted on each string end points, then the conditions for mechanical equilibrium follow from Eqs. (A12) and (A13). With the conventions shown in Fig. 10, we get

\footnotetext{
${ }^{19}$ Here the upper sign refers to string 1 and the lower sign to string 2.
}

$$
\sigma_{x}-F_{x}=0
$$

at the point $A$ and

$$
F_{x}-\sigma_{x}=0, \quad F_{r}-\sigma_{r}=0
$$

at the point $B$.

Configuration $4 \mathrm{~b}$ may be analyzed along the above lines. The only novelty is that a cusp forms at the point $C$. This happens because $C$ lies on the induced horizon and the simple root is $r_{\bar{\sigma}}=r_{v}$. The gluing conditions (A21) are satisfied by $\sigma_{x}^{(i)}= \pm \bar{\sigma}_{v}$ and $\sigma_{r}^{(i)}=0$.

Now, the angle $\theta$ at the end point $B$ is determined by

$$
\sin \theta=-\frac{\bar{\sigma}_{v}}{\bar{\sigma}}
$$

As a result, the string length $\ell$ can be written as

$$
\begin{aligned}
\ell= & r_{h} \int_{0}^{\frac{r_{v}}{r_{h}}} \frac{d \rho}{f\left(r_{h} \rho\right)} \sqrt{f\left(r_{h} \rho\right)-v^{2}}\left(\frac{\bar{\sigma}^{2}\left(r_{h} \rho\right)}{\bar{\sigma}_{v}^{2}}-1\right)^{-\frac{1}{2}} \\
& +r_{h} \int_{\nu}^{\frac{r_{v}}{r_{h}}} \frac{d \rho}{f\left(r_{h} \rho\right)} \sqrt{f\left(r_{h} \rho\right)-v^{2}}\left(\frac{\bar{\sigma}^{2}\left(r_{h} \rho\right)}{\bar{\sigma}_{v}^{2}}-1\right)^{-\frac{1}{2}} .
\end{aligned}
$$

From the formula (A10), it follows that the cusp (deviation) angle is given by

$$
\tan \gamma=\left.\frac{1}{v^{2}} \sqrt{\frac{\partial_{r} f}{2 \partial_{r} \ln \bar{\sigma}}}\right|_{r_{v}} .
$$

Clearly, nothing special happens with the conditions for force balance at the string end points, and therefore, these are also given by Eqs. (A26) and (A27).

We conclude our discussion of the double-string configurations with configuration $4 \mathrm{c}$. In this case, we use the first integral of the equation of motion to parameterize the $\sigma_{x}$ 's at the point $C$ as $\sigma_{x}^{(i)}= \pm \bar{\sigma} \sin \theta\left(r_{b}\right)$ subject to the condition $\bar{\sigma}|\sin \theta|\left(r_{b}\right)<\bar{\sigma}_{v}$. The latter guarantees a real right-hand side in Eq. (A10). As before, both $\sigma_{r}^{(i)}$,s vanish at $C$. All of this together is enough to meet the gluing conditions at $C$.

Combining the first integral and Eq. (A11), we find a useful formula for the string length along the $x$ direction

$$
\begin{aligned}
\ell= & r_{h} \int_{0}^{\frac{r_{v}}{r_{h}}} \frac{d \rho}{f\left(r_{h} \rho\right)} \sqrt{f\left(r_{h} \rho\right)-v^{2}}\left(\frac{1}{\sin ^{2} \theta} \frac{\bar{\sigma}^{2}\left(r_{h} \rho\right)}{\bar{\sigma}^{2}\left(r_{h} \nu\right)}-1\right)^{-\frac{1}{2}} \\
& +r_{h} \int_{\nu}^{\frac{r_{v}}{r_{h}}} \frac{d \rho}{f\left(r_{h} \rho\right)} \sqrt{f\left(r_{h} \rho\right)-v^{2}}\left(\frac{1}{\sin ^{2} \theta} \frac{\bar{\sigma}^{2}\left(r_{h} \rho\right)}{\bar{\sigma}^{2}\left(r_{h} \nu\right)}-1\right)^{-\frac{1}{2}} .
\end{aligned}
$$

Since in the case of interest $r_{\bar{\sigma}}>r_{v}$, we get from Eq. (A10) that the cusp angle is simply 

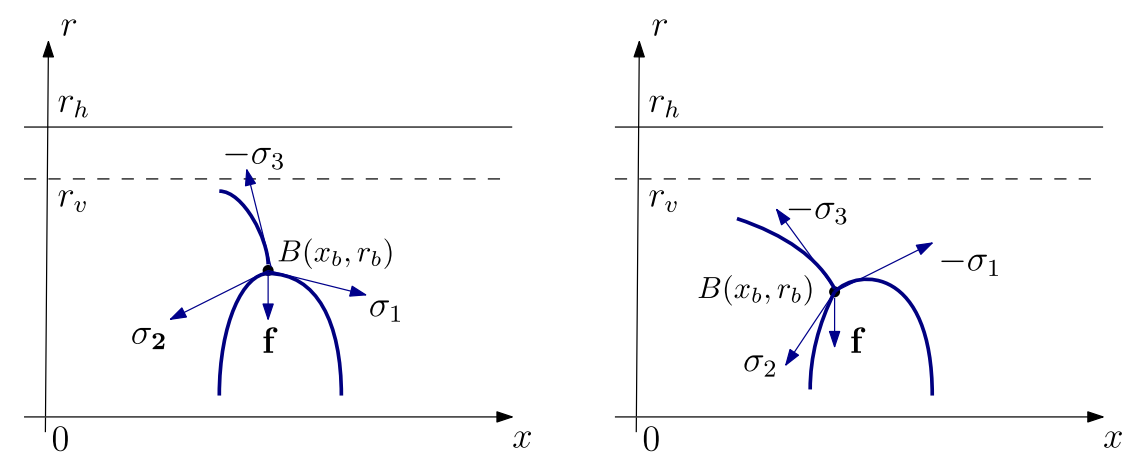

FIG. 11. Three strings meeting in a baryon vertex placed at $B$. The gravitational force acting on the vertex is directed in the downward vertical direction. In vector notation, $\boldsymbol{\sigma}=\left(\sigma_{x}, \sigma_{r}\right)$ and $\mathbf{f}=\left(0, f_{r}\right)$. (Left) The configuration is constructed from the configurations of Fig. 9. (Right) The configuration is constructed from the two configurations of Fig. 9 and one of Fig. 10.

$$
\tan \gamma=0
$$

Finally, there is no difficulty in understanding that all the conditions for mechanical equilibrium are the same as before.

\section{APPENDIX B: GLUING CONDITIONS}

The basic string configurations we have discussed in Appendix A provide the blocks for building multistring configurations. What is also needed are certain gluing conditions for strings meeting at baryon vertices (threestring junctions). Here we will describe such conditions. In doing so, we restrict ourself to the planar case so that all strings lie on the $x$-r plane.

We begin with the configuration shown in Fig. 11 on the left. The gluing conditions come from the requirement that in the variation of the total action with respect to a location of the vertex all boundary contributions cancel out. This is equivalent to a force balance condition at that vertex. Dropping all but the boundary terms at $B$, we find that the variation of the total action is

$$
\begin{aligned}
\delta \mathcal{S}= & \int d t\left(\sigma_{x}^{(1)}+\sigma_{x}^{(2)}-\sigma_{x}^{(3)}\right) \delta x_{b} \\
& +\left(\sigma_{r}^{(1)}+\sigma_{r}^{(2)}-\sigma_{r}^{(3)}+f_{r}\right) \delta r_{b}+\cdots,
\end{aligned}
$$

where the $\sigma$ 's can be read from the corresponding formulas of Appendix A. The gravitation force acting on the vertex is

$$
f_{r}=-m \partial_{r}\left(\sqrt{f-v^{2}} \mathcal{V}\right)
$$

as follows from Eq. (2.8). After a little algebra, we find

$$
\sin \theta_{1}+\sin \theta_{2}+\phi=0
$$

and

$$
\begin{aligned}
& \cos \theta_{1}+\cos \theta_{2}-\sqrt{1-\phi^{2}} \\
& +3 \kappa \frac{\sqrt{f}}{w}\left(\partial_{r} \mathcal{V}+\frac{1}{2} \mathcal{V} \partial_{r} \ln \left(f-v^{2}\right)\right)=0
\end{aligned}
$$

with $\phi=\frac{\bar{\sigma}_{v}}{\bar{\sigma}}$ and $\kappa=\frac{m}{3 \mathfrak{g}}$. These conditions determine $\theta_{1}$ and $\theta_{2}$ as functions of $r_{b}, v$, and $r_{h}$. Note that $\theta_{1}$ is negative, while $\theta_{2}$ is positive. $\phi$ takes values on the interval $[0,1]$, because $\bar{\sigma}$ decreases with increasing $r$ and $r_{b}<r_{v}$.

It is straightforward to repeat the above analysis for the configuration shown in Fig. 11 on the right. The only difference is that string 1 changes its shape from configuration 1 of Fig. 9 to one of those shown in Fig. 10. In accordance with our conventions in Appendix A, the condition (B3) holds, but Eq. (B4) now becomes

$$
\begin{aligned}
& \cos \theta_{1}-\cos \theta_{2}+\sqrt{1-\phi^{2}} \\
& -3 \kappa \frac{\sqrt{f}}{w}\left(\partial_{r} \mathcal{V}+\frac{1}{2} \mathcal{V} \partial_{r} \ln \left(f-v^{2}\right)\right)=0 .
\end{aligned}
$$

As before, these conditions determine the $\theta$ 's in terms of $r_{b}$, $v$, and $r_{h}$. It is worth noting that if string 2 changes its shape to configuration 2 of Fig. 9, then nothing happens with the form of the gluing conditions.

\section{APPENDIX C: SMALL $\nu$ EXPANSION}

Here we explore in more detail the example discussed in Sec. III. Our goal is to compute the first two Taylor coefficients of $\ell$ expanded about $\nu=0$. The other coefficients can be computed in a similar way.

As already noted in Sec. III B 4, it follows that for small $\nu$, only configuration I needs to be retained. If so, then $\ell$ is given by Eq. (3.4) with the $\theta$ 's determined from Eqs. (3.5a) and (3.5b). It is quite natural to expand

$$
\theta_{i}(\nu ; v, h)=\sum_{n=0, n \text { even }}^{\infty} \theta_{i}^{(n)} \nu^{n},
$$


and then to plug these series into the gluing equations. After some algebra, we arrive at

$$
\begin{aligned}
& \theta_{i}^{(0)}=\mp \sqrt{1-\varkappa^{2}}, \\
& \theta_{i}^{(2)}= \pm \frac{3}{2} \frac{\kappa \varkappa h}{\sqrt{1-\varkappa^{2}}}-\frac{1}{2} \frac{v}{\sqrt{1-v^{2}}} \mathrm{e}^{h \sqrt{1-v^{2}}},
\end{aligned}
$$

where $\varkappa=\frac{1}{2}(1+3 \kappa)$. The upper sign refers to $\theta_{1}$, and the lower sign to $\theta_{2}$.

Now, plugging Eq. (C2) into Eq. (3.4) and performing the integral over $\rho$, we get finally

$\ell(\nu ; v, h)=\frac{1}{\sqrt{\mathfrak{I}}} \sqrt{1-v^{2}} \sqrt{h} \nu\left(\ell^{(1)}+\ell^{(3)} h \nu^{2}+O\left(\nu^{4}\right)\right)$,

with

$$
\begin{aligned}
\ell^{(1)}=\frac{2}{3} \sqrt{1-\varkappa^{2}}{ }_{2} F_{1}\left[\frac{1}{2}, \frac{3}{4}, \frac{7}{4}, 1-\varkappa^{2}\right], \\
\ell^{(3)}=\frac{2+\varkappa-4 \varkappa^{2}}{6 \varkappa^{2} \sqrt{1-\varkappa^{2}}}\left(5_{2} F_{1}\left[-\frac{1}{2}, \frac{3}{4}, \frac{7}{4}, 1-\varkappa^{2}\right]\right. \\
\left.-\left(2+\varkappa^{2}\right)_{2} F_{1}\left[\frac{1}{2}, \frac{3}{4}, \frac{7}{4}, 1-\varkappa^{2}\right]\right) \\
-\frac{\varkappa}{\left(1-\varkappa^{2}\right)^{\frac{3}{2}}}\left(\frac{1}{\varkappa^{2}}-2+{ }_{2} F_{1}\left[-\frac{1}{4}, 1, \frac{1}{4}, 1-\varkappa^{2}\right]\right) .
\end{aligned}
$$

Here, ${ }_{2} F_{1}$ is the hypergeometric function.

One point is worthy of note. To second order in $\nu$, the length transforms according to the Lorentz contraction formula. This is a puzzling result, because there is no Lorentz invariance in the thermal medium which defines a preferred rest frame. The resolution is that such a transformation formula is violated by higher-order terms.

\section{APPENDIX D: TAYLOR SERIES OF $\sigma_{s}$}

In Sec. IV, we needed to know the coefficients of the Taylor series of $\sigma_{s}(T, \mu)$ about the point $(T, 0)$. Now we want to show that in fact these can be computed analytically. Here, as above, we restrict attention to the first two coefficients.

Following Ref. [8], we first deduce from the relations (4.2) that

$$
8 x U=9 h^{\frac{3}{2}}\left(1-Z y^{2}\right),
$$

where

$U=\mathrm{e}^{\frac{3}{2} h}-1-\frac{3}{2} h, \quad Z=\frac{9+(7+6 h) \mathrm{e}^{-2 h}-16 \mathrm{e}^{-\frac{1}{2} h}}{1-2 \mathrm{e}^{-\frac{1}{2} h}+\mathrm{e}^{-h}}$.

Here we have rescaled $T$ and $\mu$ as $T=\frac{\sqrt{3}}{\pi} x$ and $\mu=2 \mathfrak{r} \sqrt{3 \mathfrak{g}} y$. Expanding $h$ about $y=0$,

$$
h(x, y)=h_{0}(x)+h_{2}(x) y^{2}+O\left(y^{4}\right),
$$

and then inserting it into Eq. (D1), we find

$$
\left.x=\frac{9}{8} h_{0} 0^{\frac{3}{2}} U^{-1}, \quad h_{2}=\frac{2}{3} \frac{h_{0} 0 Z U}{U+h_{0} 0\left(1-\mathrm{e}^{\frac{3}{2}} h_{0} 0\right.}\right) .
$$

The first equation gives us $x$ as a function of $h_{0}$, while the second represents a recursion relation between $h_{0}$ and $h_{2}$. Note that from Eq. (D1), it follows easily that the series (D3) contains only even powers of $y$. Therefore, Eq. (4.3) is an expansion in even powers of $\mu$.

Now, it is straightforward to compute the desired coefficients. Using the expansion for $h$ and the formula for the spatial string tension (4.1), after a short computation we get

$\sigma_{s}^{(0)}=\sigma \frac{\mathrm{e}^{h_{0} 0-1}}{h_{0} 0}, \quad \sigma_{s}^{(2)}=\frac{9 h_{0} 0^{3}\left(h_{0} 0-1\right) Z \sigma_{s}^{(0)}}{128 \pi \mathfrak{r}^{2} U\left(U+h_{0} 0\left(1-\mathrm{e}^{\frac{3}{2} h_{0} 0}\right)\right)}$,

with $\sigma=\mathfrak{g} \mathfrak{g e}$. Thus, the coefficients are given parametrically by Eqs. (D4) and (D5), where $h_{0}$ is a parameter. The other coefficients can be obtained similarly, but with more effort.
[1] M. Gell-Mann, Phys. Lett. 8, 214 (1964).

[2] A. Esposito, A. Pilloni, and A. D. Polosa, Phys. Rep. 668, 1 (2017); R. F. Lebed, Phys. Rev. D 94, 034039 (2016); T. Mehen, Phys. Rev. D 96, 094028 (2017); E. J. Eichten and C. Quigg, Phys. Rev. Lett. 119, 202002 (2017).
[3] M. Döring, K. Hübner, O. Kaczmarek, and F. Karsch, Phys. Rev. D 75, 054504 (2007).

[4] F. Prino and R. Rapp, J. Phys. G 43, 093002 (2016); R. Rapp and H. van Hees, in Quark Gluon Plasma 4, edited by R. C. Hwa and X.-N. Wang (World Scientific, Singapore, 2010). 
[5] J. Casalderrey-Solana, H. Liu, D. Mateos, K. Rajagopal, and U. A. Wiedemann, Gauge/String Duality, Hot QCD, and Heavy Ion Collisions (Cambridge University Press, Cambridge, England, 2014).

[6] C. P. Herzog, A. Karch, P. Kovtun, C. Kozcaz, and L. G. Yaffe, J. High Energy Phys. 07 (2006) 013; S. S. Gubser, Phys. Rev. D 74, 126005 (2006).

[7] O. Andreev, Mod. Phys. Lett. A 33, 1850041 (2018).

[8] O. Andreev, Phys. Rev. D 94, 126003 (2016).

[9] E. Witten, J. High Energy Phys. 07 (1998) 006.

[10] O. Andreev and V. I. Zakharov, J. High Energy Phys. 04 (2007) 100.

[11] U. Gürsoy, E. Kiritsis, G. Michalogiorgakis, and F. Nitti, J. High Energy Phys. 12 (2009) 056.

[12] O. Andreev and V. I. Zakharov, Phys. Lett. B 645, 437 (2007).

[13] For pure $S U(3)$ gauge theory, the warp factor $w=\mathrm{e}^{\mathfrak{3} r^{2}}$ does the job-not outstanding, but good enough. See Refs. [7,8,14] and also O. Andreev, Phys. Rev. D 76, 087702 (2007); Phys. Rev. Lett. 102, 212001 (2009).
[14] O. Andreev, Phys. Lett. B 756, 6 (2016); Phys. Rev. D 93, 105014 (2016).

[15] H. Liu, K. Rajagopal, and U. A. Wiedemann, Phys. Rev. Lett. 98, 182301 (2007).

[16] D. Bak, A. Karch, and L. G. Yaffe, J. High Energy Phys. 08 (2007) 049.

[17] S.-J. Sin and I. Zahed, Phys. Lett. B 648, 318 (2007).

[18] A. Chamblin, R. Emparan, C. V. Johnson, and R. C. Myers, Phys. Rev. D 60, 104026 (1999).

[19] M. Döring, S. Ejiri, O. Kaczmarek, F. Karsch, and E. Laermann, Eur. Phys. J. C 46, 179 (2006).

[20] R. F. Lebed, Phys. Lett. B 749, 454 (2015); R. Zhu and C.-F. Qiao, Phys. Lett. B 756, 259 (2016).

[21] O. Andreev, Phys. Rev. D 78, 065007 (2008).

[22] B. Zwiebach, A First Course in String Theory (Cambridge University Press, Cambridge, England, 2009).

[23] P. C. Argyres, M. Edalati, and J. F. Vazquez-Poritz, J. High Energy Phys. 01 (2007) 105. 